\title{
Design of Intelligent Temperature and Humidity Controller for Tobacco Bulk Curing Barn
}

\author{
Fengzhong $\mathrm{Hu}^{1,}$, , Jinding $\mathrm{Gao}^{1, \mathrm{~b}}$, Tianxiao $\mathrm{Li}^{2, \mathrm{c}}$ \\ ${ }^{1}$ College of Information Science and Engineering, Hunan International Economics University, \\ Changsha, Hunan, 410205, China \\ ${ }^{2}$ Hunan Jiutian Technology Co. Ltd., Changsha, Hunan, 410205, China \\ ahugo126@126.com, bjdgao@126.com, ctianxiao@163.com
}

\begin{abstract}
Keywords: Tobacco Bulk Curing Barn, Temperature Control, Humidity Control, Intelligent Control Abstract. Aimed at the temperature and humidity nonlinear variation and coupling phenomena in tobacco bulk curing barn, an intelligent temperature and humidity controller is proposed. The controller using a microcomputer and fuzzy decoupling control technique can meet the requirements of temperature and humidity variation according to the predetermined technology curves, realized tobacco curing overall automation, thereby improved the quality of fluecured tobacco. The principle and composition of the controller are presented in this paper. The control method can be applied to other temperature and humidity control field when it is changed in some sort, has popularization and application value.
\end{abstract}

\section{Introduction}

Tobacco bulk curing barn is the specialized apparatus for tobacco bulk curing, the quality of fluecured tobacco depends on the temperature control and humidity control in tobacco curing process. During the curing process, temperature and humidity interaction and nonlinearity is more serious, and affect each other. In the heating process, the moisture content of tobacco leaves increases, during the dehumidification process, the temperature can also be affected because of the introduction of outdoor air. This coupling phenomenon of conventional control method is difficult to meet the requirements. For this purpose, an intelligent temperature and humidity controller based on fuzzy decoupling control is designed. The controller can detect temperature and humidity of tobacco room and control corresponding actuators automatically according to the predetermined technology curve, realized tobacco curing overall automation, improved the quality of flue-cured tobacco.

\section{Control principle [1,2]}

The control principle by using micro controller unit and fuzzy control theory is shown in Fig. 1, according to the temperature error and humidity error detected, on the basis of fuzzy control rule established in ROM of the micro controller unit, to determine the control output, then, through the decoupling coefficient by experience determined to realize fuzzy decoupling control.

In figure $1, \mathrm{~S}_{\mathrm{t}}$ and $\mathrm{T}_{\mathrm{t}}$ respectively represent the set temperature and real temperature, $\mathrm{E}_{\mathrm{t}}, \mathrm{E}_{\mathrm{t}}{ }^{\prime}$ are temperature deviation and deviation rate; $S_{h}$ and $T_{h}$ respectively represent the set humidity and real humidity, $\mathrm{E}_{\mathrm{h}}, \mathrm{E}_{\mathrm{h}}$ ' are humidity deviation and deviation rate; $\mathrm{C}_{\mathrm{t}}, \mathrm{C}_{\mathrm{h}}$ are respectively for control output. If using the NB, NM, NS, ZO, PS, PM, PB respectively represent each of these variables is negative big, negative medium, negative small, zero, positive small, positive medium, positive big, by the bulk curing barn temperature and humidity control experience, can be summed up experience fuzzy control rules as shown in table 1, table 1 shows, a total of 49 fuzzy control rule exist, for example, if $\left(E_{t}=N B\right)$ AND $\left(\mathrm{E}_{\mathrm{t}}{ }^{\prime}=\mathrm{NB}\right)$ THEN $\left(\mathrm{C}_{\mathrm{t}}=\mathrm{PB}\right)$.

In this controller, given fuzzy subset of $E_{t}, E_{h}, E_{t}, E_{h}, C_{t}, C_{h}$ associated with $\{N B, N M, N S, Z O$, PS, PM, PB \}, given quantization level of input variable $\mathrm{E}_{\mathrm{t}}, \mathrm{E}_{\mathrm{h}}, \mathrm{E}_{\mathrm{t}}{ }^{\prime}, \mathrm{E}_{\mathrm{h}}$ ' associated with 13 grade, that is $\{-6,-5,-4,-3,-2,-1,0,1,2,3,4,5,6\}$. Choose uniform triangle function to describe the variables 
membership function corresponding to its field, as shown in Fig. 2, we can obtain the equation (1) and equation (2) according to the rules of fuzzy inference synthesis.

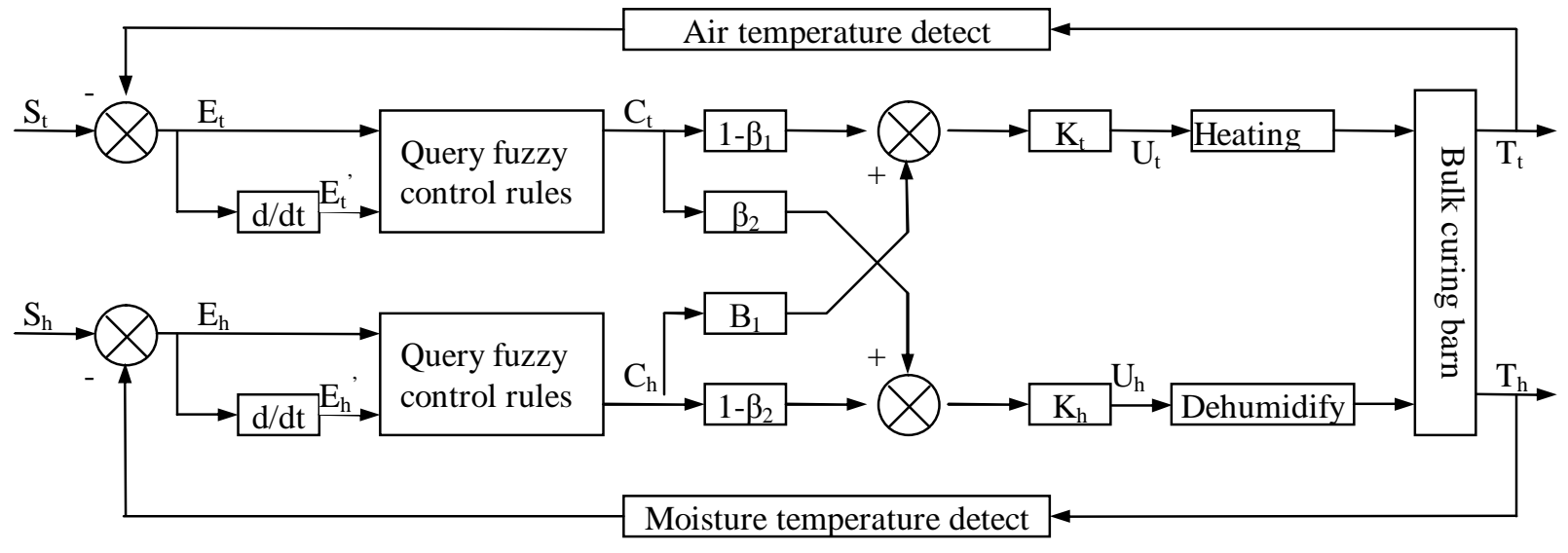

Fig. 1 Fuzzy decoupling control principle

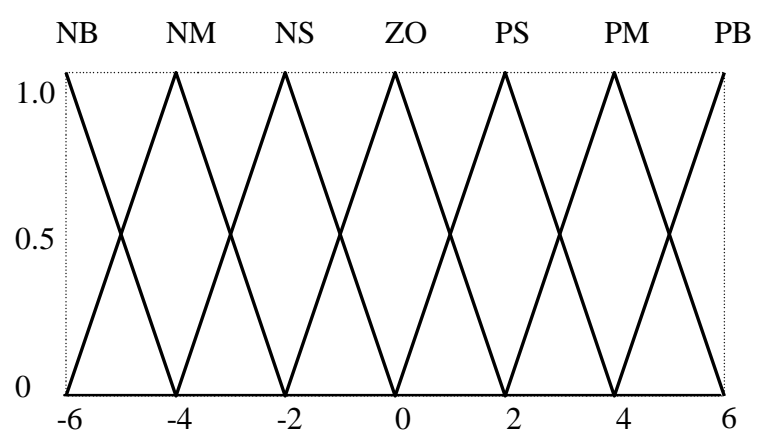

Fig. 2 Fuzzy membership functions for the controller
Table 1 Fuzzy control rules

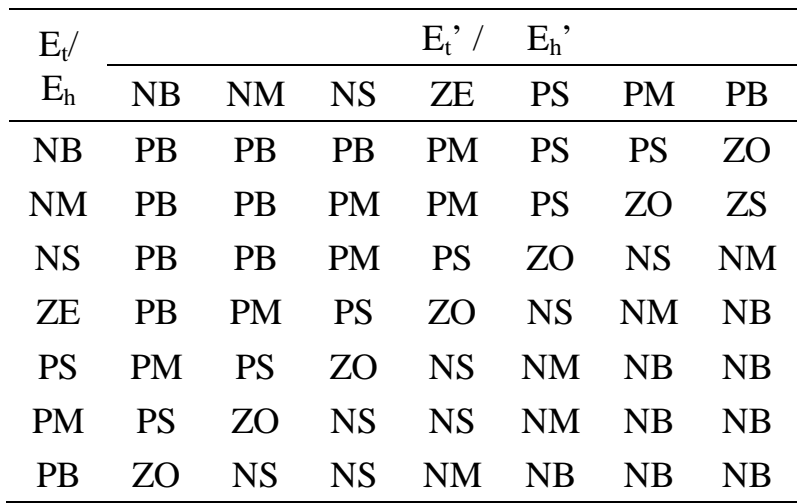

$$
\begin{aligned}
& C_{t}=\left(E_{t} \times E_{t}{ }^{\prime}\right) \times R_{t} \\
& C_{h}=\left(E_{h} \times E_{h}{ }^{\prime}\right) \times R_{h}
\end{aligned}
$$

Through introducing the decoupling coefficient $\beta_{1}$ and $\beta_{2}$ further realize the decoupling control.

$$
\begin{array}{ll}
\mathrm{U}_{\mathrm{t}}=\mathrm{K}_{\mathrm{t}}\left[\left(1-\beta_{1}\right) \times \mathrm{C}_{\mathrm{t}}+\beta_{1} \times \mathrm{C}_{\mathrm{h}}\right] & \left(\beta_{1}=0 \sim 1\right) \\
\mathrm{U}_{\mathrm{h}}=\mathrm{K}_{\mathrm{h}}\left[\left(1-\beta_{2}\right) \times \mathrm{C}_{\mathrm{h}}+\beta_{2} \times \mathrm{C}_{\mathrm{t}}\right] & \left(\beta_{2}=0 \sim 1\right)
\end{array}
$$

The actual value of $\beta_{1}$ and $\beta_{2}$ need to be determined by experiment, specifically, in the curing process from the beginning of 0 increases ceaselessly, when temperature and humidity fluctuations of the hour, the values of $\beta_{1}$ and $\beta_{2}$ are optimal decoupling coefficient.

\section{The Controller Hardware Design}

The controller is composed of a controller main board, an operation panel, sensors interface, actuators drive module interface, power supply module etc., its composition principle as shown in Fig.3 on the left dashed box. The actuators include a circulating fan, a heating furnace fan and air inlet damper mounting in the bulk curing barn. The control system can achieve heating up and steadying temperature through controlling oxygen quantity of coal burning by adjusting rotate speed of heating furnace fan, the control system can keep humidity smooth in curing barn by controlling quantity of air entering in fact. In addition, through adjusting rotate speed of circulating fan, decreases difference in temperature between top and bottom of curing barn, achieves fundamental leveling of temperature. The controller can effectively control running states of actuators, these is the reason that ensure the baking quality of tobacco. 


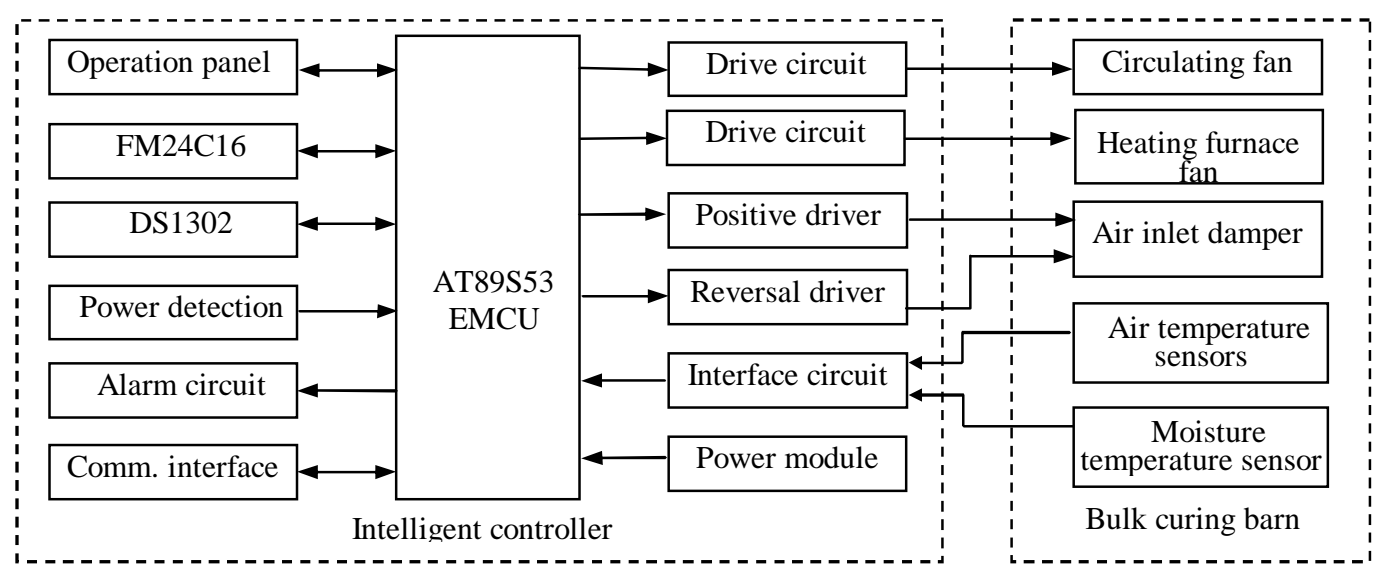

Fig. 3 Tobacco bulk curing barn controller block diagram

The controller main board circuit as shown in Fig.4. AT89S53 is a low-power, high-performance CMOS 8-bit microcomputer with $12 \mathrm{~K}$ bytes of downloadable Flash, 256 bytes of RAM, 32 I/O lines, programmable watchdog timer, three 16-bit timer/counters, a six-vector two-level interrupt architecture, a full duplex serial port, on-chip oscillator, and clock circuitry. FM24C16 is nonvolatile memory for storing the set temperature and humidity curves, expert curves and baking process data. Clock chip DS1302 is used to provide the corresponding time of baking process to MCU. Power detect circuit is used for detection of power-down to switch to a battery power supply. Using a pair of DS18B20 sensor to detect the temperature and humidity of tobacco in bulk curing barn, DS18B20 is a programmable resolution 1-wire digital thermometer, has $\pm 0.5^{\circ} \mathrm{C}$ accuracy from $-10^{\circ} \mathrm{C}$ to $+85^{\circ} \mathrm{C}$, is very suitable for long distance data transmission.

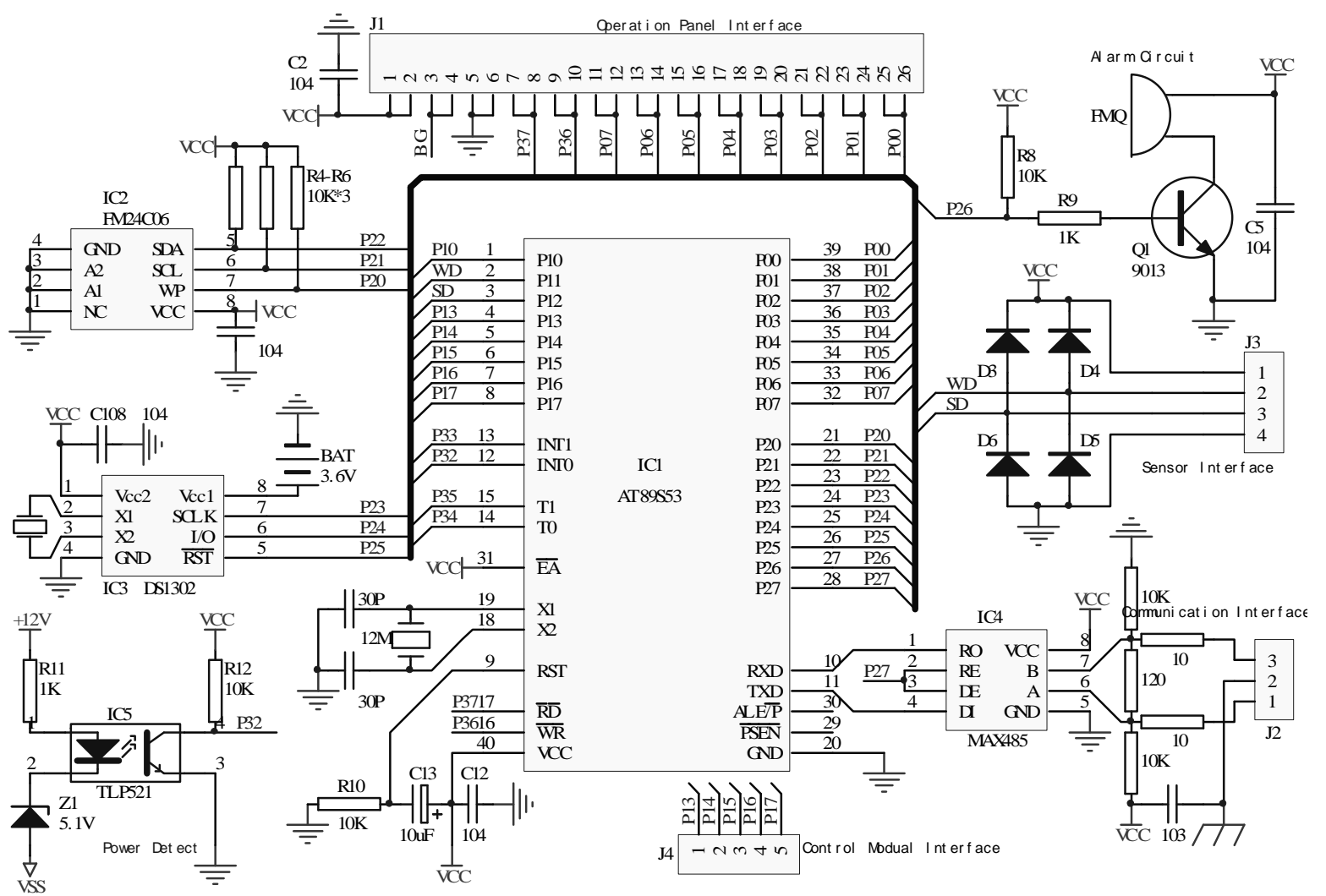

Fig. 4 The controller main board circuit

\section{The controller program design}

The controller work flow is: before baking, firstly choose a specialist technology curve stored in controller or set up a technology curve according to process requirements through the operation panel, and then start baking. During the baking process, the temperature and humidity controller control 
actuators based on the detection and target temperature and humidity, around a preset process curve automatic regulation. When the air temperature or moisture temperature exceeds the set value of $\pm 2.0^{\circ} \mathrm{C}$, the controller alarm and display alarm code. Here are the main program flowchart fig. 5 and fuzzy decoupling control algorithm flowchart fig. 6 .

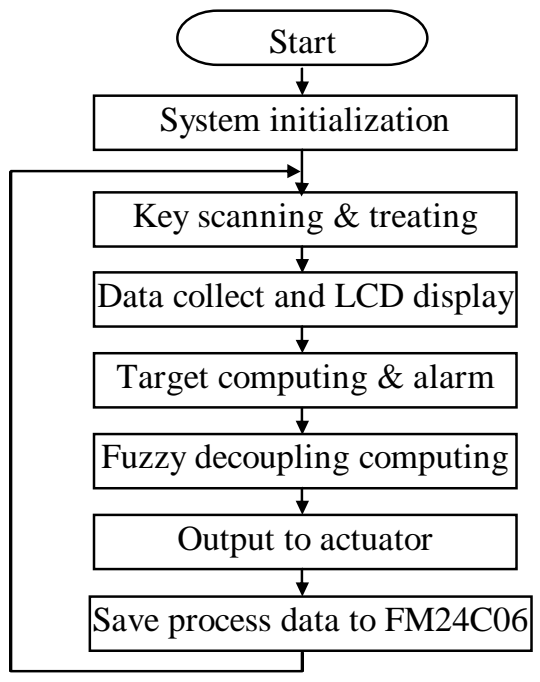

Fig. 5 Flowchart of main program

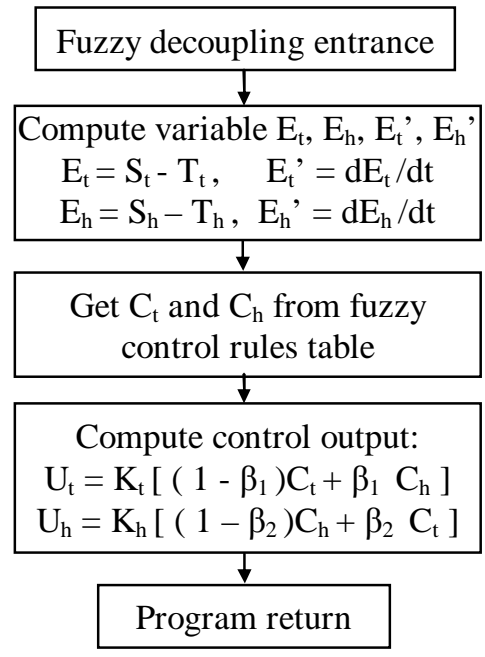

Fig. 6 Flowchart of fuzzy decoupling algorithm

\section{Conclusions}

Through the practical test in tobacco bulk curing barn, the controller based on the above design has $\pm 1.0^{\circ} \mathrm{C}$ air/moisture temperature control accuracy from $20^{\circ} \mathrm{C}$ to $80^{\circ} \mathrm{C}$, the temperature-humidity coupling phenomena in tobacco baking process is prevented, fluecured tobacco in color, moisture, elastic and other aspects of quality are improved. In addition, compared with the traditional control methods, it has a short time to reach the set temperature, small steady-state temperature fluctuation, sensitive reaction, strong anti-jamming ability. The control method introduced in this paper can be applied to porcelain baking, crops baking etc., has popularization and application value.

\section{Acknowledgements}

This work was supported by Scientific Research Fund of Hunan Provincial Education Department under Grant No. 15A106.

\section{References}

[1] J.Y. Zhang and X.N. Tang: Journal of Agricultural Mechanization Research Vol. 8 (2008), p.65

[2] Z. Kovacic: Fuzzy Controller Design-Theory and Applications (CRC Press, USA. 2006).

[3] J.K. Liu: Intelligent control (Publishing House of Electronics Industry, Beijing 2005).

[4] E. Karakas: Engineering Application of Artificial Intelligence Vol.16 (2003), p.717

[5] Q.S. Pang, P.F. Fu, and X.Q. Zhong: Proceedings of 2011 International Conference on Electronics, Communication and Control (ICECC) Vol. 4 (2011), p. 2065

[6] H.X. Li and H.B. Gatland: IEEE Transactions on Fuzzy Systems Vol. 5 (1997), p.145

[7] B. Hu, G.K.I. Mann and R.G. Gosine: IEEE Trans. on Fuzzy Systems Vol. 7 (1999), p.521

[8] Information on http://www.taylorandfrancis.com 